\title{
Curious case of acute encephalopathy in a 64-year- old male
}

\begin{abstract}
A 64-year-old male presents with acute encephalopathy and upon further evaluation, he was found to have lymphadenopathy in the neck and parotid region. Subsequent lumbar puncture (LP) revealed the presence of blast cells in the CSF concerning for leukemic meningitis. A lymph node biopsy was consistent with CD5 positive DLBCL. He was started on chemotherapy with Hyper-CVAD and intrathecal methotrexate, improving his clinical condition but he was lost to follow up.

Diffuse large B-cell lymphoma (DLBCL) is the most common type of non-Hodgkin lymphoma (NHL). Diagnosis is based on symptoms and excisional lymph node biopsy with immunotyping. It is extremely important to have a lymph node biopsy if suspicious for lymphoma because of its aggressive nature. Indicators for such include CD5 positivity and extranodal involvement. The risk factors for CNS involvement in DLBCL include high International Prognostic Index (IPI) score, bone marrow involvement, and extranodal involvement such as bone, lung and testis.

The purpose of the report is to emphasize the importance of ruling out lymphoma when diffuse lymphadenopathy is present in the setting of encephalopathy; the potential of aggressive behavior of this disease including involvement of the CNS; and discussing the overall social determinants of health for this patient resulting in delayed diagnosis and lack of an optimal treatment plan to be carried out.
\end{abstract}

Keywords: acute encephalopathy, diffuse large B-Cell lymphoma, international prognostic index, R-CHOP, hyper-CVAD
Volume I 2 Issue 2 - 202I

\author{
S Blake O'Brien,' Joseph R Malhis, 'Alex \\ Belote,' Brooke Brander ${ }^{2}$ \\ 'Internal Medicine Resident PGY-2, University of Arkansas for \\ Medical Sciences Northwest Regional Campus, USA \\ ${ }^{2}$ Highlands Oncology Group, USA
}

Correspondence: Joseph R Malhis, DO, MA, Internal Medicine Resident PGY-2, University of Arkansas for Medical Sciences Northwest Regional Campus, Fayetteville, AR, USA, Emailymalhis@gmail.com

Received: April 29, 202I | Published: May II, 202 I

\section{Background//ntroduction}

Diffuse Large B-cell Lymphoma (DLBCL) is the most common type of Non-Hodgkin's lymphoma, seen in $25 \%$ of cases. ${ }^{1}$ Categorization is heterogeneous with the different subtypes including primary DLBCL of the mediastinum (thymic), primary DLBCL in the CNS and associated with chronic inflammation. ${ }^{1}$ Patients typically present with an enlarging nodal mass that is located in the neck, abdomen, mediastinum but can appear anywhere. ${ }^{2}$ Common symptoms include "B" symptoms like fever greater than $100.4 \mathrm{~F}$, weight loss greater than $10 \%$ of body weight over six months and drenching night sweats. ${ }^{3}$ Roughly $40 \%$ of patients will have extranodal involvement and $1 / 3$ of patients will have systemic symptoms. ${ }^{4}$ Diagnosis is made with excisional tissue biopsy on lymph nodes to evaluate morphology and immunotyping. The biopsy may reveal diffuse effacement of the normal structure as well as atypical lymphoid cells having prominent nucleoli and basophilic cytoplasm. ${ }^{4}$ Typical markers for B-cells are cluster of differentiation or CD19, CD20, CD22 and CD79a. CD 30 is seen in $25 \%$ of cases and it is the anaplastic variant. Prognosis is generally good. ${ }^{5}$ However, it is rare to find CD 5 variant on staining and that is the worst prognosis and considered to be the most aggressive. ${ }^{6}$

For those who have an aggressive form of DLBCL, the international Prognostic Index (IPI) is used to predict outcome for patients. It assesses the patient's age, stage III/IV disease, elevated lactate dehydrogenase (LDH) level, Eastern Cooperative Oncology Group (ECOG) status greater than or equal to 2, and more than one extranodal site. ${ }^{7}$ Patients presenting with stage III or IV disease will have multiple nodes both sides of the diaphragm with splenic involvement. See diagram below. ${ }^{8}$
Malignant transformation can occur with other forms of nonHodgkin's lymphoma. About 30\% with follicular lymphoma can develop DLBCL. This tends to be aggressive and worse prognosis. ${ }^{4}$ Richter transformation is seen with CLL. First line treatment for an aggressive form of DLBCL is RCHOP which is a combination of 5 drugs (Rituximab, Cyclophosphamide, Doxorubicin, Vincristine and Prednisone). Alternatives include hyper-CVAD is similar to R-CHOP. Hyper-CVAD part A includes Cyclophosphamide, doxorubcin, vincristine and dexamethasone and part $\mathrm{B}$ includes and methotrexate and cytarabine (part B). Methotrexate is used for CNS penetration along with leucovorin rescue. For those who relapse, autologous HSCT and anti-CD 19 CAR-T $^{4}$ can be used. Those with double hit DLBCL would do better with Hyper-CVAD than R-CHOP.

\section{Case report}

This is a 64-year-old man with an unremarkable past medical history that presented with encephalopathy after being found unresponsive by family. Per history provided by the family, the patient has been malnourished and had 20-lb weight loss in the past few weeks. Family also reports that the patient uses alcohol and methamphetamine daily. Initial differential diagnosis included toxicmetabolic encephalopathy due to drug use, meningoencephalitis, meningitis, and leptomeningeal metastasis. On previous admission for community acquired pneumonia, a chest CT was performed, which showed diffuse lymphadenopathy of the head and neck with no further work-up done at that time. During present admission, the patient became severely agitated, and Critical Care Medicine was consulted for sedation and intubation. Once his airway was protected, Oncology was consulted for work-up of lymphadenopathy, and Surgery was 
consulted for lymph node biopsy. Additionally, Neurology was consulted for work-up of encephalopathy and lumbar puncture (LP) was performed. LP showed elevated WBC with normal protein and glucose as well as blast cells in CSF. Lymph nodes biopsied by surgery returned positive for CD5+ diffuse large B-Cell lymphoma (DLBCL), which is the most common type of Non-Hodgkin Lymphoma (NHL). Oncology believed that the patient would not survive hospitalization without treatment for lymphoma. Thus the patient was started on Hyper-CVAD part B which includes high dose methotrexate to ensure CNS penetration. However, the patient's treatment course was complicated by Streptococcus pneumoniae bacteremia based on blood cultures collected. Intravenous antibiotics slowly improved the patient's condition, and the encephalopathy resolved. While the
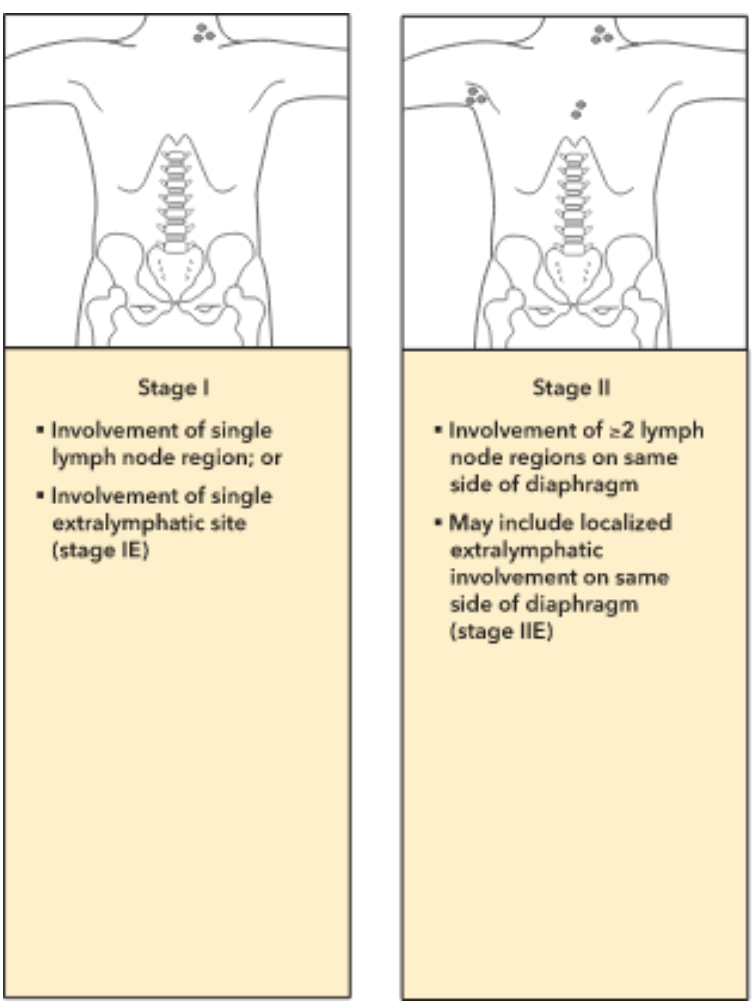

\section{Discussion}

There were multiple social and medical nuances in this gentleman's case. Due to the encephalopathic presentation caused by the CNS involvement of the lymphoma, a history was difficult to obtain without a discussion with the family. With his Strep pneumo bacteremia, the initial concern for his encephalopathy was infectious in nature. However, that is but one of many variables. There was a similar case reported in 2017 for which a patient with history of CNS involvement in DLBCL presented with acute encephalitis and he had symptoms concerning for meningitis. With that report, it was elucidated that infectious and non-infectious causes must be ruled out ${ }^{9}$ especially with a patient who cannot provide clear history. He also has a history of drug use with his urine drug screen positive for methamphetamine. So infectious encephalopathy and altered mentation from drug use and poor medical compliance made the initial diagnosis difficult When the patient was no longer altered and able to answer questions, there was concern for potential elder abuse from his children. Case management was consulted for aid in contacting Adult Protective Services (APS). However, he left against medical advice prior to this occurring. At the time, it was unclear whether he departed of his own accord or due to pressure from family. patient was beginning to show improvement clinically, he decided to leave against medical advice. His prognosis was poor with a score of $4 / 5$ on International Prognostic Index Calculator (IPI) for DLBCL, which translates to an overall survival rate of $55 \%$. Poor prognostic indicators include age $>60$, Ann Arbor Stage III-IV, ECOG score $>2$, Serum LDH $>1 \mathrm{x}$ normal, and $>1$ extranodal site present. This patient followed up with Oncology as an outpatient and did agree to admission again for more chemotherapy He once again left AMA following his treatment, but he did continue to keep appointments as an outpatient. While his functional status did initially improve, he eventually began to deteriorate after treatment. He unfortunately succumbed to his disease about six months after it was initially discovered.
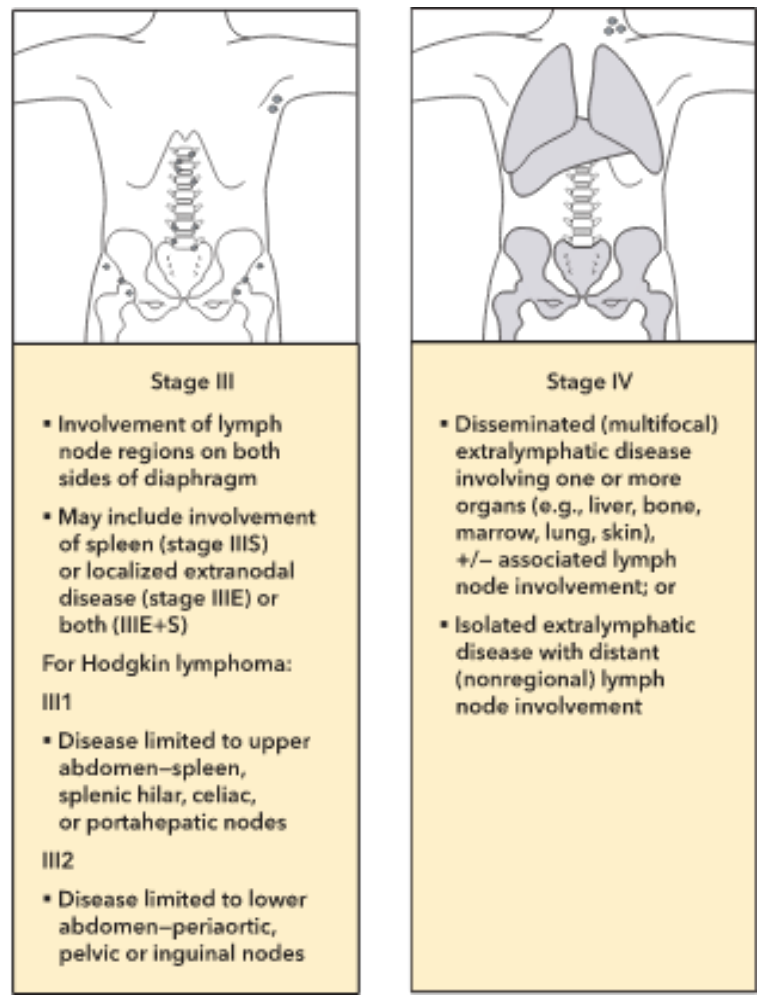

After further research on his previous medical history, it was discovered that two weeks before his admission at our facility, he was admitted for community acquired pneumonia at a different facility, and imaging showed mediastinal lymphadenopathy. However, he did not have further work up while admitted and it was the hospital's understanding that he was to be followed up as an outpatient. Following discussions with the family, it is unclear why this never occurred. The patient did have a supportive significant other that offered to take him to follow-up appointments and chemotherapy treatments, so followup was scheduled with Oncology.

Oncology decided to use a hyper-CVAD treatment regimen rather than an R-CHOP regimen for few reasons. Due to the CNS involvement of the lymphoma, intrathecal methotrexate would be required as part of the R-CHOP regimen, but our hospital facility does not offer intrathecal methotrexate, and it was unclear whether the patient was going to adhere to a treatment schedule. Therefore, he was initially started on hyper-CVAD because of its CNS penetration. Hyper-CVAD can be utilized for double-hit DLBCL, which this patient did not have; however, this was unknown until after the patient's departure AMA. The part B aspect of Hyper-CVAD has the methotrexate and high dose cytarabine which can provide some CNS penetration. However, 
the total amount of methotrexate in this regimen is not completely sufficient for full CNS penetration. ${ }^{10}$ Intrathecal methotrexate will still be necessary for optimal treatment. For this reason, at a future follow-up, it was decided that an Ommaya reservoir would be placed for outpatient administration of intrathecal methotrexate. This would require fewer trips to the hospital as the patient lived about an hour away and transportation was already an issue. With his aggressive high grade B-cell lymphoma, if the intrathecal methotrexate does not work and he relapses, autologous stem cell transplantation would be another option. ${ }^{11}$ However, with his history and the delay in treatment, he may not have been an eligible candidate but it is a discussion he can have with his significant other.

Functionally, the patient improved significantly. He was awake, alert, mentally clear and ambulating on his own. His significant other was bringing him to his treatment and follow up appointments almost regularly. He initially was not expected to survive hospitalization. This case shows that persistence, empathy, understanding, and creativity in diagnosis and treatment can improve the quality of life of even the sickest patients.

\section{Conclusion}

Diffuse large B-cell Lymphoma is a very aggressive malignancy that has a poor prognosis if left untreated. Patient care is a two-way street in that any physician can help and provide what is best for the patient if said patient is willing to comply and follow through. Truly investigating patient social history is important. Having discussions about and offering resources are essential to overall patient care. Keeping this in mind could potentially help patients similar to the one presented here.

\section{Acknowledgments}

None.

\section{Conflicts of interest}

The authors declare there is no conflict of interests regarding the publication of this paper.

\section{References}

1. Morton LM, Wang SS, Devesa SS, et al. Lymphoma incidence patterns by WHO subtype in the United States, 1992-2001. Blood. 2006;107(1):265-276

2. Hui D, Proctor B, Donaldson J, et al. Prognostic implications of extranodal involvement in patients with diffuse large B-cell lymphoma treated with rituximab and cyclophosphamide, doxorubicin, vincristine, and prednisone. Leuk Lymphoma. 2010;51(9):1658-1667.

3. Armitage JO, Weisenburger DD. New approach to classifying nonHodgkin's lymphomas: clinical features of the major histologic subtypes. Non-Hodgkin's Lymphoma Classification Project. J Clin Oncol. 1998;16(8):2780-2795.

4. Lymphoid Malignancies: Hematology and Oncology. MKSAP: ACP 18.

5. Slack GW, Steidl C, Sehn LH, et al. CD30 expression in de novo diffuse large B-cell lymphoma: a population-based study from British Columbia. Br J Haematol. 2014;167(5):608-617.

6. Katzenberger T, Lohr A, Schwarz S, et al. Genetic analysis of de novo CD5+ diffuse large B-cell lymphomas suggests an origin from a somatically mutated CD5+ progenitor B cell. Blood. 2003;101(2):699-702.

7. Sehn LH, Brian Berry, Mukesh Chhanabhai, et al. The revised International Prognostic Index (R-IPI) is a better predictor of outcome than the standard IPI for patients with diffuse large B-cell lymphoma treated with R-CHOP. Blood. 2007;109(5):1857-1861.

8. Ann Arbor Staging System for Hodgkin and non-Hodgkin lymphoma. Reprinted with permission from Skarkin, A. The Atlas of Diagnostic Oncology, 3rd Edition. Philadelphia, PA: Mosby; 2002.

9. Moonga SS, Liang K, Cunha BA. Acute Encephalitis in an Adult with Diffuse Large B-Cell Lymphoma with Secondary Involvement of the Central Nervous System: Infectious or Non-Infectious Etiology? J Clin Med. 2017;6(12):117.

10. Kevin H, Panjic EH, Valla K, et al. "How to Decide which DLBCL Patients should Receive CNS Prophylaxis." Cancer Network: Home of the Journal ONCOLOGY; 2018.

11. Skrabek P, Assouline S, Christofides A, et al. Emerging therapies for the treatment of relapsed or refractory diffuse large B cell lymphoma. Curr Oncol. 2019;26(4):253-265. 